\title{
A study to assess the distribution of cystic and solid hepatic lesionson contrast enhanced helical computed tomography
}

\author{
Rani $\mathbf{S}^{1}$, Khantal $\mathbf{N}^{2}$, Tripathi $\mathbf{P}^{3}$ \\ ${ }^{1}$ Dr. Sonika Rani, Senior Resident, ${ }^{2}$ Dr. Nitin Khantal, Assistant Professor, ${ }^{3}$ Dr. Poorva Tripathi, Senior Resident, All \\ authors are affiliated with Department of Radiodiagnosis Chirayu Medical College and Hospital, Bhopal, MP, India.
}

Corresponding Author: Dr. Nitin Khantal, Department of Radiodiagnosis, Chirayu Medical College and Hospital, Bhopal (M.P.). E-mail: drkhants10@gmail.com

\begin{abstract}
Introduction: Focal liver lesions are defined as solid or liquid-containing masses foreign to the normal anatomy of the liver that may be told apart from the latter organ using imaging techniques. Material and Methods: This prospective study was done in the Department of Radiodiagnosis and imaging at Bhopal Medical Centre, Bhopal, Madhya Pradesh, India. A total of 100 patients who were referred to our department with strong clinical suspicion of focal liver lesion and those diagnosed by ultrasonography underwent multiphasic contrast enhanced CT evaluation of abdomen using Single slice Spiral CT scanner from March 2010 to May 2012. Results: The majority of lesions appeared as solid lesions on CT comprising $63 \%$ of lesions. The remaining were cystic lesions comprising 37\% of cases.Among the cystic lesions, the most common CT diagnosis was Simple Cyst Seen in $49 \%$ of cases. The other diagnosis was liver abscess in $30 \%$ and hydatid cyst in $21 \%$. Among the solid lesions, the most common CT diagnosis was that of metastases seen in $49 \%$ of cases. The next common lesions seen were HCC seen in $22.5 \%$ of cases and hemangioma seen in $19.5 \%$ of cases. Discussion: Simple cysts are diagnosed by the presence of a well defined intra hepatic mass of fluid attenuation, no perceptible wall or internal septations and no enhancement. Among solid lesions, haemangiomas were diagnosed by the presence of a hypodense lesion with peripheral nodular enhancement of arterial attenuation with centripetal fill in. Conclusion: Most of the lesions evaluated were solid compared to cystic. Among cystic lesions, simple cyst was the most common abnormality seen in around half the cases followed by liver abscess and hydatid cyst. Among the solid lesions, the most common abnormality detected was metastases seen in around half the cases followed by HCC and hemangioma.
\end{abstract}

Keywords:Cystic lesion, solid hepatic lesion, Contrast enhanced helical computed tomography.

\section{Introduction}

Focal liver lesions are defined as solid or liquidcontaining masses foreign to the normal anatomy of the liver that may be told apart from the latter organ using imaging techniques [1]. They may be benign, malignant or metastatic in origin. Commonly encountered benign lesions include pyogenic liver abscess, focal nodular hyperplasia, simple cyst, hydatid cyst and hemangiomas.

Malignant lesions include Hepatocellular carcinoma, Intrahepatic Cholangiocarcinoma. Metastatic lesions include secondaries from colon, lung, breast, stomach, pancreas, prostate, etc. [2]. The objectives of liver

Manuscript received: $14^{\text {th }}$ May 2018

Reviewed: $24^{\text {th }}$ May 2018

Author Corrected: $30^{\text {th }}$ May 2018

Accepted for Publication: $4^{\text {th }}$ June 2018 imaging in oncology are the detection of the liver disease, the characterization of liver lesions, the staging of neoplasms, the evaluation of biliary ductal status, the evaluation of treatment response, and the assessment of vascular anatomy for surgical planning and chemotherapy pump placement [3].

CT offers the best spatial resolution and the ability to study the entire liver in a single breath-hold. Recent technological advances in CT technology, such as helical CT and multidetector row helical CT, have further improved the performance of CT scanners in terms of speed of acquisition, resolution, and the ability to image the liver during various phases of contrast enhancement more precisely than was possible previously [4]. 


\section{Original Research Article}

Recent advances in CT provide higher spatial and temporal resolution for the evaluation of liver tumor hemodynamics, while also providing three-dimensional or four-dimensional imaging for treatment planning. Perfusion CT provides quantitative information about arterial perfusion in $\mathrm{HCC}$, allowing the evaluation of tumor angiogenesis and response to therapy $[5,6]$.

There is insufficient diagnostic performance for both the early detection and the characterization of small liver lesions even with computed tomography (CT) and magnetic resonance (MR) imaging techniques. As such, there is a need to improve on morphology-based CT and MR imaging using contrast agents for the early detection and characterization of hepatic disease [7].

\section{Material and Methods}

This prospective study was done in the Department of Radiodiagnosis and imaging at Bhopal Medical Centre, Bhopal, Madhya Pradesh, India. A total of 100 patients who were referred to our department with strong clinical suspicion of focal liver lesion and those diagnosed by ultrasonography underwent multiphasic contrast enhanced CT evaluation of abdomen using Single slice Spiral CT scanner from March 2010 to May 2012.

\section{Inclusion criteria}

- Patients referred to the Radio diagnosis Department of Bhopal Medical Centre, Bhopal with strong clinical suspicion of focal lesion of liver including those with primary malignancy elsewhere.

- Patients already diagnosed with focal liver lesion by ultrasonography

\section{Exclusion criteria}

- Patients with diffuse liver diseases

- Patients with mass lesions infiltrating the liver from outside the liver

- Patients with traumatic injury to liver
Procedure- After obtaining the written consent from all the participants under the study, detailed history of the patient including signs and symptoms, detailed physical examination, biochemical investigations and radiological investigations which included chest $\mathrm{x}$-ray and ultrasonography of the abdomen were recorded.

The liver was viewed in non-contrast enhanced phase, arterial phase, portal venous phase and delayed phase in axial, saggital and coronal sections and any abnormality was identified. When multiple lesions are noted the most representative lesion or the largest of the lesions was taken into consideration.

When different types of lesions were identified in the same person representative lesions of each type was considered. The following characteristics of the lesions were noted.

- The number of lesions

- The segmental location of the lesion

- The size and shape of the lesion

- The presence of calcification/ septa/ internal nodules

- The wall/ thickness of wall/sharpness of contour

- Homogenous/heterogenous

- Presence/absence of enhancement

- Pattern of enhancement in arterial, portal venous and delayed phases

- Potency of vessels

- Surrounding hepatic parenchyma

- Other specific features.

Follow up of all patients were done either with biopsy, aspiration, surgical correlation, follow-up ultrasonography to look for the stability of the lesion or additional investigations like MRI/nuclear scintigraphy.

The final diagnosis was made. Then results obtained were compared with the multiphasic contrast enhanced helical CT imaging findings and tabulated.

\section{Results}

The present study was carried out in the Department of Radiodiagnosis Bhopal Medical Centre, Bhopal, Madhya Pradesh.

A total of 100 patients were included which were referred to our department with history of focal liver lesions. Contrast Enhanced Multiphasic Computed Tomography was performed and evaluated for the underlying focal liver lesion.

In our study, majority of patients belonged to the age group 30-39 Years (29\%) and males constituting $55 \%$ of cases. The most common clinical presentation was that of pain in abdomen seen in $57 \%$ of cases. The second most common presentation was that of history of malignancy elsewhere seen in $36 \%$. 
Table No.-1: Frequency of cystic lesions and solid lesions.

\begin{tabular}{|c|c|c|}
\hline Appearance & No. Of cases & \% of cases \\
\hline Cystic & 37 & $37 \%$ \\
\hline Solid & 63 & $63 \%$ \\
\hline Total & $\mathbf{1 0 0}$ & $\mathbf{1 0 0}$ \\
\hline
\end{tabular}

The majority of lesions appeared as solid lesions on CT comprising 63\% of lesions. The remaining were cystic lesions comprising $37 \%$ of cases.

Table No.-2: Cystic lesions distribution.

\begin{tabular}{|c|c|c|c|}
\hline Lesion & Criteria & No. of cases & \% of cases \\
\hline Simple cyst & homogenous, imperceptible wall, no enhancement & 18 & $49 \%$ \\
\hline Hydatid cyst & Perceptible wall, enhancement, daughter cysts or \\
& membrane & 8 & $21 \%$ \\
\hline Liver abscess & thick, irregular wall ring enhancement, surrounding edema & 11 & $30 \%$ \\
\hline Total & & $\mathbf{3 7}$ & $\mathbf{1 0 0 \%}$ \\
\hline
\end{tabular}

Among the cystic lesions, the most common CT diagnosis was Simple Cyst Seen in $49 \%$ of cases. The other diagnosis was liver abscess in $30 \%$ and hydatid cyst in $21 \%$.

Table No.-3: Solid lesions distribution.

\begin{tabular}{|c|c|c|c|}
\hline Lesions & Criteria & $\begin{array}{l}\text { No. of } \\
\text { cases }\end{array}$ & $\%$ of cases \\
\hline Hemangioma & $\begin{array}{c}\text { hypodense with peripheral nodular enhancement with } \\
\text { centripetal fill in }\end{array}$ & 12 & $19.5 \%$ \\
\hline FNH & $\begin{array}{l}\text { hypodense with homogenous arterial enhancement with } \\
\text { hypodense central cleft }\end{array}$ & 1 & $1.5 \%$ \\
\hline Focal fat & $\begin{array}{c}\text { hypodense, no enhancement, adjacent to falciform } \\
\text { ligament, no mass effect }\end{array}$ & 2 & $3 \%$ \\
\hline Hcc & $\begin{array}{c}\text { heterogenous hypodense with enhancement in arterial } \\
\text { phase, washout in delayed phase }\end{array}$ & 14 & $22.5 \%$ \\
\hline Cholangio carcinoma & $\begin{array}{l}\text { hypodense ill defined margins with delayed prolonged } \\
\text { enhancement }\end{array}$ & 1 & $1.5 \%$ \\
\hline IHE & $\begin{array}{l}\text { hypodense ill defined lesion with arterial enhancement } \\
\text { and isodense in delayed phase }\end{array}$ & 1 & $1.5 \%$ \\
\hline Hepatoblastoma & $\begin{array}{c}\text { hypodense lesion with calcification and mixed } \\
\text { enhancement }\end{array}$ & 1 & $1.5 \%$ \\
\hline Metastases & $\begin{array}{c}\text { hypo-iso-or hyper, homo-or heterogenous lesions with } \\
\text { variable enhancement }\end{array}$ & 31 & $49 \%$ \\
\hline Total & & 63 & 100 \\
\hline
\end{tabular}

Among the solid lesions, the most common CT diagnosis was that of metastases seen in $49 \%$ of cases. The next common lesions seen were HCC seen in $22.5 \%$ of cases and hemangioma seen in $19.5 \%$ of cases.

\section{Discussion}

A cystic lesion is defined as one having an average attenuation coefficient between 0 and $20 \mathrm{HU}$ [8]. Among the liver lesions solid lesions constituted the majority with $63 \%$ of cases and cystic lesions constituting a minority of $37 \%$ of cases.
Simple cysts are diagnosed by the presence of a well defined intra hepatic mass of fluid attenuation, no perceptible wall or internal septations and no enhancement [8]. Among the patients with simple cysts, one case of multiple liver cysts (more than 10) 


\section{Original Research Article}

associated with polycystic kidney Disease having multiple cortical cysts in the kidney was observed. Hydatid cysts are diagnosed by the presence of a well defined lesion of fluid attenuation with a perceptible wall which may undergo enhancement. Calcifications or daughter cysts or membranes may be present [8]. Among these, calcification was seen in 6/8 (75\%) of cases and daughter cysts were seen in $4 / 8(50 \%)$ of cases.

Liver abscesses are diagnosed by the presence of a well defined lesion with thick, irregular wall, ring enhancement and surrounding edema [9]. But one case of cystic metastases from Gall Bladder mass was wrongly diagnosed as liver abscess (false positive).

Among solid lesions, haemangiomas were diagnosed by the presence of a hypodense lesion with peripheral nodular enhancement of arterial attenuation with centripetal fill in $[10,11]$.

FNH was diagnosed as a hypo/ iso dense lesion with homogenous arterial enhancement and a hypodense central cleft corresponding to the scar [12]. One case was wrongly diagnosed as metastases (false negative) and it had Hyper-A-A pattern.

Focal fat was diagnosed by the presence of a hypohypo-hypo lesion showing no enhancement, located adjacent to the falciform ligament and without any mass effect.

HCC was diagnosed by the presence of a heterogenous hypodense mass with hyper enhancement in the arterial phase with or without abnormal internal vessels and washout in venous phase [13]. One case of regenerative nodule in cirrhotic liver and a case of hemangioma were wrongly diagnosed as HCC (false positive).

Cholangiocarcinoma was diagnosed by the presence of a hypodense ill defined lesion with delayed and prolonged enhancement [14].

IHE was identified by the presence of solitary/ multiple hypodense lesions with arterial enhancement becoming isodense in the delayed phase. One case was seen and diagnosed correctly using these criteria. Multiple lesions were seen distributed throughout the liver. Associated cutaneous hemangiomas were also seen. Hepatoblastoma was diagnosed by the presence of a large hypodense lesion with calcification and mixed pattern of enhancement. This was similar to the study conducted by Dachman et al [15].
Thus the differentiation of cystic and solid lesions using Contrast Enhanced Multiphasic Computed Tomography in our study shows high accuracy and also helps in further characterization of the lesions based on the morphological and enhancement characteristics.

\section{Conclusion}

Most of the lesions evaluated were solid compared to cystic. Among cystic lesions, simple cyst was the most common abnormality seen in around half the cases followed by liver abscess and hydatid cyst. Among the solid lesions, the most common abnormality detected was metastases seen in around half the cases followed by HCC and hemangioma.

The accuracy of Contrast Enhanced Multiphasic Computed Tomography in detecting and characterizing focal liver lesions is high and it should be considered in the imaging work up of any patient with focal liver lesions.

This helps in guiding further management of these patients avoiding unnecessary investigations and work up for the diagnosis as there is a high incidence of benign pathologies in the liver.

\section{Funding: Nil, Conflict of interest: None Permission of IRB: Yes}

\section{References}

1. Pons F, Llovet JM. Approaching focal liver lesions. Rev Esp Enferm Dig. 2004 Aug;96(8):567-73; 573-7.

2. Kumar PB, Hegde P, Kumar BNK et al. A Comparative Study of Ultrasound and CT findings in Focal Liver Lesions. Int J Biol Med Res. 2014; 5(3): 4362-4369.

3. Sica GT, Ji H, Ros PR. CT and MR imaging of hepatic metastases. AJR Am J Roentgenol. 2000 Mar; 174 (3): 691-8. DOI: 10.2214/ajr.174.3.1740691

4. Francis IR, Cohan RH, Mc Nulty NJ et al. Multidetector CT of the liver and hepatic neoplasms: effect of multiphasic imaging on tumorconspicuity and vascular enhancement. AJR Am J Roentgenol. 2003 May; 180 (5): 1217-24.

5. Zhu AX, Holalkere NS, Muzikansky A, Horgan $\mathrm{K}$, Sahani DV. et al. Early antiangiogenic activity of bevacizumab evaluated by computed tomography perfusion scan in patients with advanced hepatocellular carcinoma. doi: 10.1634/theoncologist.2007-0174. 


\section{Original Research Article}

6. Ippolito D, Sironi S, Pozzi M, Antolini L, Ratti L, Alberzoni C, Leone EB, Meloni F, Valsecchi MG, Fazio F. et al. Hepatocellular carcinoma in cirrhotic liverdisease: functional computedtomography with perfusion imaging in the assessment of tumor vascularization. doi: 10.1016/j.acra.2008.02.005.

7. Semelka RC, Martin DR, Balci C, et al. Focal liver lesions: comparison of dual-phase CT and multisequence multiplanar MR imaging including dynamic gadolinium enhancement. J MagnReson Imaging. 2001 Mar;13(3):397-401.

8. Murphy BJ, Casillas J, Ros PR, Morilloet al. The CT appearance of cystic masses of the liver.10. 1148/ radiographics.9.2.2538868

9. J P Heiken, P J Weyman, J K Lee, et al. Detection of focal hepatic masses: prospective evaluation with CT, delayed CT, CT during arterial portography, and MR imaging. doi.org/ 10. 1148/ radiology.171.1.2538862.

10. van Leeuwen MS, Noordzij J, Feldberg MA, et al. Focal liver lesions: characterization with triphasic spiral CT.DOI: 10.1148/radiology.201.2.8888219
11.Nino-Murcia M, Olcott EW, Jeffrey RB Jr, Lamm RL, et al.Focal liver lesions: pattern-based classification scheme for enhancement at arterial phase CT. DOI: 10. 1148 /radiology.215.3.r00jn03746

12. Lee Joseph KT, Stuart S. Sagel, Robert J. Stanley et al. Computed body Tomography with MRI correlation 2004.

13. Fernandez MP, Redvanly RD. Primary hepatic malignant neoplasms.Radiol Clin North Am. 1998 Mar; 36 (2): 333-48.

14. Loyer EM, Chin H, Du Brow RA, et al. Hepatocellular carcinoma and intrahepatic peripheral cholangiocarcinoma: enhancement patterns with quadruple phase helical CT--a comparative study. DOI: 10. 1148/ radiology.212.3.r99se32866

15. Dachmann Abraham H, Lichtenstein JE, Friedman AC et al. Infantile hemangioendothelioma of the liver. A radiologic- pathologicclinical correlation. AJR 1983; 140; 10911096

\section{How to cite this article?}

Rani S, Khantal N, Tripathi P. A study to assess the distribution of cystic and solid hepatic lesionson contrast enhanced helical computed tomography. Int J Med Res Rev 2018;6(05):266-270. doi:10.17511/ijmrr. 2018.i05.05. 